ARTICLE

\title{
Investigating the effect of habitat modification on chronic stress in deer mice: a preliminary study
}

\author{
Andreas Eleftheriou' \& Angela D. Luis ${ }^{2}$ \\ ${ }^{1}$ Wildlife Biology Program, The University of Montana, Missoula, MT, USA; \\ ${ }^{2}$ Department of Ecosystem and Conservation Sciences, The University of Montana, Missoula, MT, USA
}

\begin{abstract}
Anthropogenic habitat modification can lead to chronic stress in wildlife. This can result in immunosuppression and higher disease prevalence. Chronically stressed individuals typically have elevated baseline GCs and decreased body condition. GCs are called FGMs when excreted in feces and can be used to noninvasively evaluate stress in free-ranging wildlife. In the deer mouse (Peromyscus maniculatus)-SNV system, SNV prevalence is higher in deer mice at peridomestic settings, which are human-modified habitats. This is problematic because SNV causes a fatal disease in humans, and thus the higher SNV prevalence may lead to higher risk of infection for humans. In our study, we hypothesized that SNV prevalence would be higher in deer mice at human-modified habitats due to chronic stress. To test our hypothesis, we compared two stress measures (i.e., baseline FGMs and body condition scores) in deer mice from one peridomestic and one sylvan grid over 2 months. Captured deer mice were tagged, weighed, sexed and sampled for feces and blood and were evaluated for reproductive status and body condition before release. Blood samples were analysed for SNV antibodies, and fecal samples were evaluated for FGMs. We found higher deer mouse numbers at the sylvan grid. There were no differences in baseline FGM levels between peridomestic and sylvan populations. However, peridomestic deer mice had overall lower body condition. Given the low SNV prevalence across both grids, we were unable to examine potential correlations between SNV prevalence and chronic stress. Regardless, we conclude that deer mice at human-modified habitats may not be chronically stressed, which may suggest that higher SNV prevalence at peridomestic settings may not be the result of chronic stress. Although we did find that peridomestic deer mice had lower body condition, this may not have been related to chronic stress because there were no differences in baseline FGMs. Longer studies with more site replication are needed to validate and expand on our findings. Our preliminary study adds to the existing body of knowledge that examines relationships between stress physiology and disease prevalence in human-modified environments.
\end{abstract}

\section{Introduction}

Human-modified habitats can have negative effects on wildlife health. For example, anthropogenic habitat modification can lead to chronic stress, which can affect immunity and disease prevalence (Brearley et al. 2013). Chronically stressed individuals may have elevated baseline GCs, a dysregulated acute stress response (Dantzer et al. 2014) and decreased body condition (Cyr \& Romero 2009). Effects on immunity from chronic stress can result in higher susceptibility to infection (Bradley $\&$ Altizer
2007). GCs are steroid hormones (Easterbrook \& Klein 2008), which are called FGMs when excreted in feces (Touma \& Palme 2005). FGM measurement is considered a non-invasive means for stress evaluation in freeranging wildlife (Millspaugh \& Washburn 2004).

North American deer mice (Peromyscus maniculatus; hereafter deer mice) are distributed over much of North America (Baker 1968), are habitat generalists (Wywialowski 1987) and may enter human dwellings in both rural (Hopkins et al. 2002) and urban sites (Kuenzi et al. 2000). They are the wildlife reservoir for SNV, which 
is a hantavirus transmissible to humans (Childs et al. 1994). SNV causes a chronic infection in deer mice (Mills et al. 1999), but they do not seem to be clinically affected from infection (Easterbrook \& Klein 2008). However, a few studies demonstrated that infected male deer mice may suffer from lower survival (Luis et al. 2012) and weight gains (Douglass et al. 2007). Human infection with SNV can cause HCPS, which can have a mortality of up to $40 \%$ (Bi et al. 2008). SNV exposure in humans may occur more often at peridomestic settings (Armstrong et al. 1995) and appears to be positively correlated with deer mouse density (Calisher et al. 2011).

There is evidence for higher SNV prevalence in deer mice at peridomestic settings compared to sylvan settings (Kuenzi et al. 2001). This higher prevalence could be attributed to various causes such as higher deer mouse density and longer survival of SNV in the external environment. Additionally, SNV prevalence may be higher if deer mice at peridomestic settings are more chronically stressed and, consequently, more immunosuppressed than their sylvan counterparts. Such immunosuppression may make individuals more likely to become infected with SNV.

In this study, we hypothesized that SNV prevalence would be higher in deer mice at human-modified habitats because of chronic stress. SNV prevalence varies widely over space and time (e.g., Mills et al. 1999), and unfortunately, the overall SNV prevalence during our study was too low to make inferences about habitat modification and SNV prevalence. Therefore, in this paper, we concentrated on how habitat modification is related to chronic stress, which has implications for SNV spread in deer mice. To test this, we conducted a preliminary mark-recapture study at two sites (one sylvan and one peridomestic) at a cattle ranch in western Montana over 2 months (November and December) in 2016. To evaluate chronic stress, we used two measures: (1) baseline FGM levels and (2) body condition score. Although we wanted to use the stress response to an acute stressor (i.e., trap confinement) as an additional measure, we were unable to do so.

\section{Materials and methods}

Live trapping of deer mice was conducted as previously done (e.g., Kuenzi et al. 2001) with some modifications to accommodate for FGM evaluation. Two 1-ha grids were established (one peridomestic and one sylvan). Each grid had 100 trap stations $10 \mathrm{~m}$ apart in a $10 \times 10$ array. These grids were located at a cattle ranch near Drummond, MT. The peridomestic grid was located adjacent to the ranchers' house and barn. It consisted mostly of grasses and was commonly visited by the ranchers' cattle and dogs. The sylvan grid was located near an occasionally used dirt road and was at least $500 \mathrm{~m}$ away from any houses or barns. This was sagebrush habitat with much less disturbance from cattle and dogs. However, horses were observed near this grid. Deer mice were live trapped overnight for three consecutive nights once in November and once in December in 2016. Sherman traps (H. B. Sherman, Tallahassee, FL) were baited with peanut butter and oats and supplied with polyester bedding. Traps were opened around dusk and checked approx. 4 hours later to evaluate baseline stress, given that FGMs from feces collected within 4 hours of trap setup are less likely to be influenced by trap-induced stress (Harper \& Austad 2001). We returned deer mice to their respective traps so we could evaluate their stress response to an acute stressor (i.e., overnight trap confinement). Therefore, deer mice were processed twice: once at night and another around dawn. All non-target individuals were released without further processing, and traps were removed from grids after each trapping session.

\section{Animal processing}

At the initial check (approx. 4 hours after setup), traps with animals were taken to a central location for processing. Fecal samples were collected from deer mice, which were tagged with metal ear tags (National Band and Tag Co., Newport, KY) before they were returned to their respective traps. At the last check (around dawn after overnight trap confinement), deer mice were weighed using a spring scale and evaluated for sex, mass, ectoparasites and reproductive status. We also evaluated each individual deer mouse for a body condition score (see Ullman-Culleré and Foltz [1999] for details on method). Age was estimated from mass as previously described (Fairbairn 1977). After all necessary sample collection was complete, deer mice were released on site.

\section{Sample collection}

Fecal samples were collected and frozen. We avoided feces that were contaminated with urine or excessive peanut butter. Blood samples were collected with heparinized microcapillary tubes from the retroorbital capillary sinus after the administration of topical anaesthesia (1-2 proparacaine drops; Akorn, Inc., Lake Forest, IL) on the chosen eye. Blood samples were then stored and frozen. Appropriate cautionary guidelines were used such as protective gloves, safety goggles and HEPA-filtered half face respirators, to avoid accidental human infection with hantavirus (Mills et al. 1995). Appropriate caution was taken to avoid unnecessary injury and distress to small mammals during field procedures. All animal handling procedures were approved by the University of Montana's IACUC. 


\section{Stress evaluation}

Fecal samples were frozen at $-80{ }^{\circ} \mathrm{C}\left(-112^{\circ} \mathrm{F}\right)$ before laboratory analysis. We heat-inactivated fecal samples at approx. $63^{\circ} \mathrm{C}\left(145.4^{\circ} \mathrm{F}\right)$ for 1 hour in a biosafety cabinet to inactivate any infectious SNV (Mills, Emory University, Atlanta, GA, personal communication) and for an additional hour to reach constant, dry weight. Dried feces were then ground into powder, and $20( \pm 5) \mathrm{mg}$ of powder was weighed out for extraction. We added $0.5 \mathrm{ml}$ of $80 \%$ methanol into powdered samples to extract FGMs. Next, the samples were vortexed for 30 minutes at $1500 \mathrm{rpm}$ and centrifuged for 20 minutes at $2500 \mathrm{rpm}$ (approx. $390 \times \mathrm{g}$ ). The supernatants were then frozen at $-80{ }^{\circ} \mathrm{C}\left(-112^{\circ} \mathrm{F}\right)$. Prior to being assayed, supernatants were diluted 1:40, 1:80 or 1:160 with assay buffer. As corticosterone is the main GC in Peromyscus maniculatus (Bradley \& Terman 1981), we quantified FGMs using a corticosterone enzyme immunoassay by following manufacturer instructions (Arbor Assays, Ann Arbor, MI). Immunoassays were read at $450 \mathrm{~nm}$ and $650 \mathrm{~nm}$. This immunoassay has been previously validated for use in deer mice (Eleftheriou et al. 2020).

We evaluated chronic stress in two ways: (1) baseline FGM levels and (2) body condition scores. Ideally, we would also evaluate the stress response to an acute stressor (i.e., overnight trap confinement). However, we were unable to do so because for this evaluation we could only use individuals that were not trapped previously, so that trap confinement could represent a novel stressor. Because several deer mice were trapped at the peridomestic grid the month prior (October) but not ear tagged, we were unsure of individuals' trapping histories.

\section{Evaluation for SNV antibodies}

We evaluated blood samples for the presence of SNV antibodies using an established ELISA (Schountz et al. 2007).

Statistics: All data analyses were done using $\mathrm{R}$ ( $\mathrm{R}$ Development Core Team 2009). We used R packages "lattice" (Sarkar 2008) and "ggplot2" (Wickham 2009) to generate figures. We used two sample t-tests to examine differences in baseline FGM levels between peridomestic and sylvan populations after ensuring data normality. Data were normalized using natural log transformation. However, we used Wilcoxon rank sum tests with continuity correction to test for differences in body condition scores between grids because these data were not normal even after various transformations. Because we had many individuals lose their ear tags across months that had to be retagged, we chose to analyse our data separately by month and compare stress measures at the population level to avoid committing pseudoreplication.
We chose not to statistically evaluate the data as a function of age and sex because the peridomestic population was too small to allow for this, and the sylvan population was mostly composed of adult males. We determined densities at grids for each month by calculating the number of unique individuals caught. We chose an $\alpha \leq 0.05$ to denote statistical significance.

\section{Results}

In November, we trapped a total of 13 deer mice at the peridomestic grid and 32 at the sylvan grid. In December, we caught six peridomestic deer mice and 20 sylvan deer mice. Hence, numbers at the peridomestic grid were consistently lower compared to the sylvan grid. Adult males comprised most of the individuals at the sylvan grid across both months (Fig. 1). We had several traps that were moved from their original locations at the peridomestic grid due to disturbance from cattle. There were a few trap locations as well that had missing traps from the peridomestic grid, most likely due to human error during trap placement. These factors may have had some influence on our evaluation of deer mouse numbers at this grid.

Baseline FGM levels were not significantly different between peridomestic and sylvan deer mice across November $(t=-1.15, p=0.27)$ or December $(t=-0.54$, $p=0.61$ ) (Fig. 2). However, we found significant differences in body condition scores between peridomestic and sylvan deer mice in November $(W=100.5, p=0.01)$ and December ( $W=11.5, p=0.01$ ) (Fig. 3). Overall, sylvan deer mice had higher body condition scores than peridomestic deer mice. We only identified two SNV-infected deer mice (both adult males), which were captured at the sylvan grid in December. The intra-assay and inter-assay coefficients of variation for the corticosterone enzyme immunoassays were less than 15\%.

\section{Discussion}

In this short-term study, we found that there were fewer deer mice at the peridomestic setting compared to the sylvan. Contrary to our initial expectations, peridomestic and sylvan individuals had similar baseline FGM levels. Nevertheless, as we expected, peridomestic deer mice had poorer body condition scores than sylvan deer mice, suggestive of chronic stress. However, our combined findings do not clearly support our hypothesis that deer mice would be chronically stressed in human-modified habitats. Although body condition scores were lower in the peridomestic population, this may reflect the effect of an environmental factor instead of physiological stress, 


\section{Demographic groups across month and grid type}

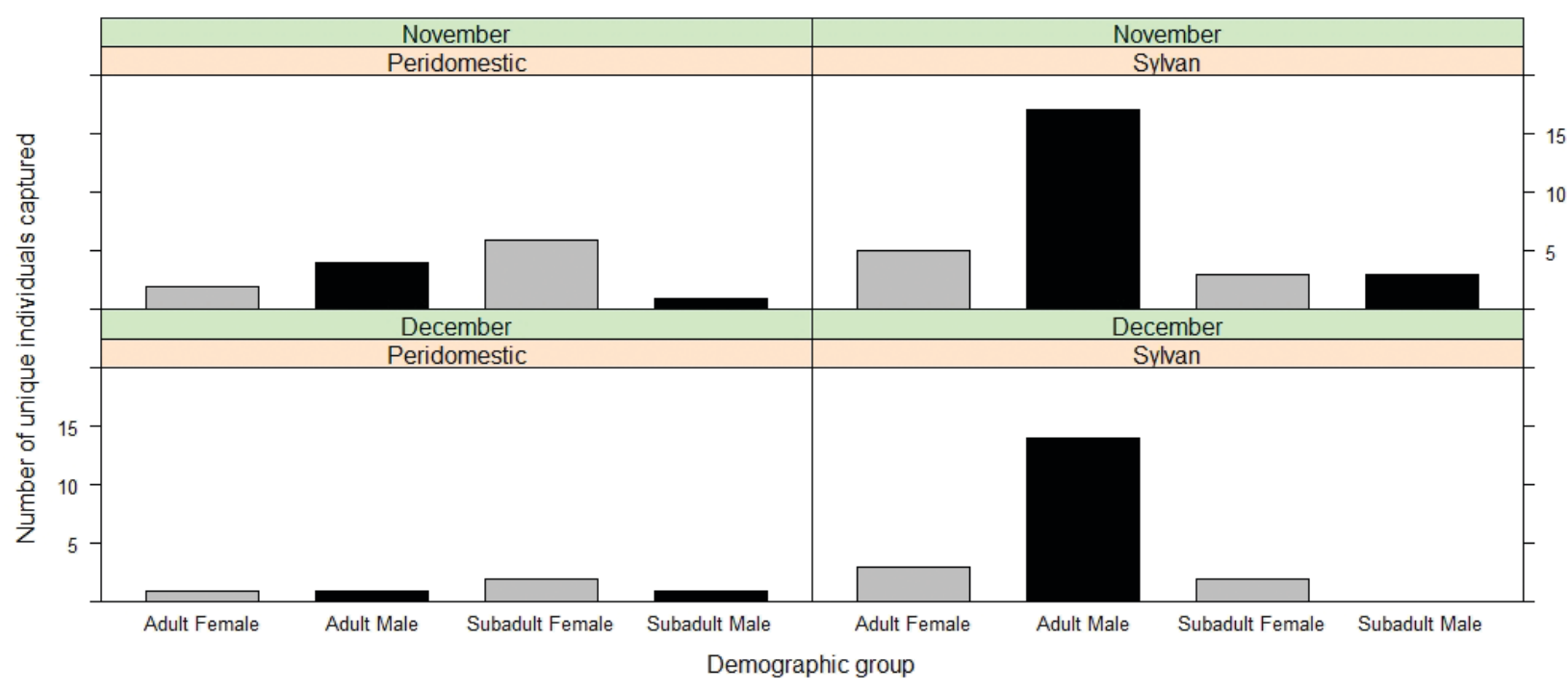

Fig. 1 Bar chart of the number of unique individuals captured (by age and sex). Numbers were lower at the peridomestic grid in November and December compared to the sylvan grid. Adult males dominated numbers of deer mice captured at the sylvan grid over both months.

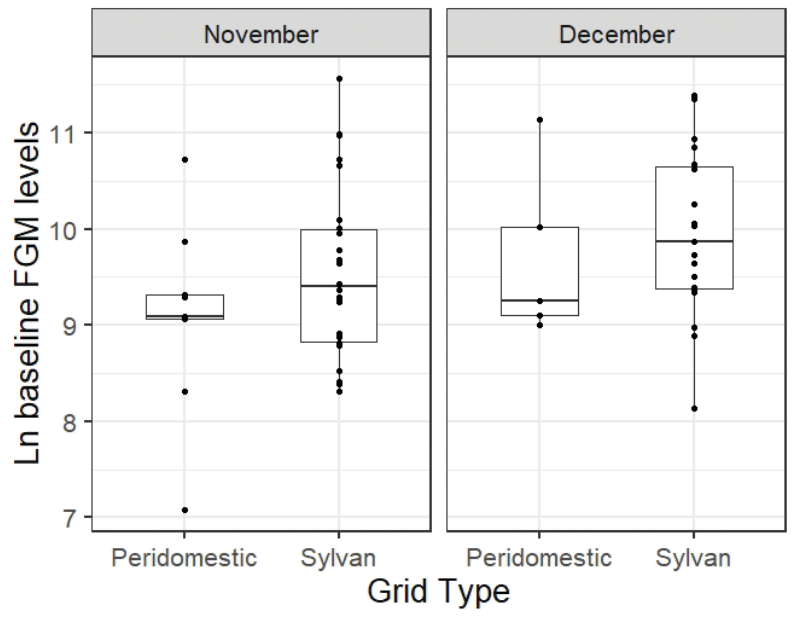

Fig. 2 Boxplots of natural log baseline FGM (stress) levels of deer mice per grid type overlaid by all individual data points. Baseline FGM levels were evaluated from feces collected approx. 4 hours after trap setup. Boxplots display the median (line), 25-75\% interquartile range (boxes) and the full range (whiskers).

given that baseline FGM levels were similar between populations. It is also possible that we saw similar FGM levels between peridomestic and sylvan populations because anthropogenic habitat modification is well tolerated by habitat generalist species such as deer mice and, thus, is not considered a chronic stressor (Brearley et al. 2013). Because our study is not replicated, we cannot accurately state why the differences in body condition scores

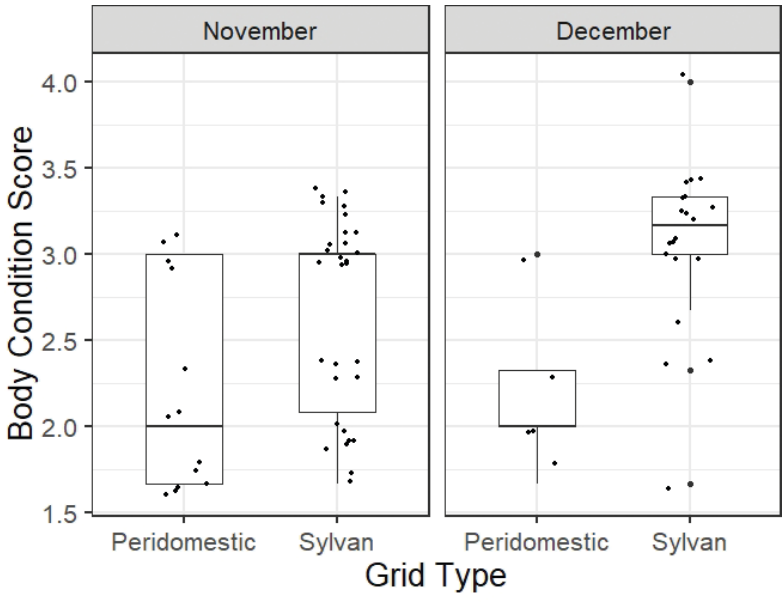

Fig. 3 Boxplots of body condition scores of deer mice per grid type overlaid by all individual data points. Peridomestic deer mice had overall, lower body condition than sylvan deer mice. Boxplots display the median (line), 25-75\% interquartile range (boxes) and the full range (whiskers).

exist between populations from the two different grids. Importantly, our study is very preliminary, and further studies across time and space are needed to validate and expand on our findings.

Although some studies found a negative relationship between stress levels and body condition (e.g., Pokharel et al. 2017), others (e.g., Mumby et al. 2015) found a positive relationship. We found that peridomestic individuals had similar baseline FGM levels but lower body 
condition compared to sylvan. These discordant results could be explained in one of two ways. First, animals that are chronically stressed for an extended time period can experience downregulation of GCs, resulting in lower FGM levels (Buscha \& Hayward 2009). However, we cannot evaluate this since we did not track FGM levels over a longer time interval. Second, as already mentioned, it is possible that peridomestic deer mice were not chronically stressed but had poorer body condition scores because of poor habitat quality and reduced food availability independent of stress physiology. We did not measure food availability so we cannot comment if this were the case. Moreover, if we were to follow these populations over a longer time scale and expose them to a pharmacological stressor (e.g., ACTH), we could examine if there were differences in stress responses between peridomestic and sylvan populations. If peridomestic individuals are indeed chronically stressed, we would expect that their response to a pharmacological stressor would be more blunted compared to sylvan individuals. It is important to note that several fecal samples were too small or too contaminated to allow for FGM evaluations. Hence, our sample sizes for FGM levels were lower compared to body condition scores. Therefore, the absence of differences using FGM levels could also be due to a smaller sample size.

We found lower deer mouse numbers at our peridomestic site compared to our sylvan site. Kuenzi et al. (2001) found the opposite pattern, but they included more grids over a longer time span than our study and trapped deer mice within human-made structures as well (e.g., buildings). Hence, we may have seen this pattern as an artifact of our study's short-time interval because we did not trap within human structures at the peridomestic grid. However, limited time and available resources necessitated for this type of study design. Lower deer mouse numbers may be suggestive that at this peridomestic grid, deer mice may not be able to thrive as well compared to the sylvan grid. Similarly, Rakotoniaina et al. (2016) found that the densities of two lemur species were lower at more disturbed habitats, although their stress levels and body condition did not differ from individuals in less disturbed habitats. They concluded that although no effects were seen on the lemurs' general health status, habitat disturbance still negatively impacted lemur fitness because densities were lower in more disturbed habitats.

In conclusion, we found that deer mice may not be chronically stressed in human-modified habitats, which suggests that chronic stress may not lead to higher SNV prevalence in peridomestic settings. However, additional studies of longer duration and more site replication are needed to validate and expand on our study's preliminary findings. It is crucial that we continue to examine relationships between disease prevalence and stress physiology because wildlife is now increasingly being exposed to various stressors, mostly of anthropogenic origin, such as habitat modification. Our study adds to the growing body of knowledge that examines chronic stress in wildlife within an anthropogenic disturbance context.

\section{Acknowledgements}

The authors would like to thank the ranchers for allowing us access to their land where this study took place. We would also like to thank the many volunteers who assisted with this study in the laboratory and field. The authors would also like to thank Dr. A. Kuenzi for her general advice and providing us with traps, Drs. L. Eby and C. Breuner for their general advice and Dr. Graham for statistical advice.

\section{Funding}

The authors acknowledge funding from the National Science Foundation under Grant No. 1836793, the National Wildlife Rehabilitators Association, and the American Society of Mammologists.

\section{References}

Armstrong L.R., Zaki S.R., Goldoft M.J., Todd R.L., Khan A.S., Khabbaz R.F., Ksiazek T.G. \& Peters C.J. 1995. Hantavirus pulmonary syndrome associated with entering or cleaning rarely used, rodent-infested structures. The Journal of Infectious Diseases 172(4), 1166. doi: 10.1093/ infdis/172.4.1166.

Baker R.H. 1968. Habitats and distribution. In J.A. King (ed.): Biology of Peromyscus (Rodentia). Pp. 98-126. Stillwater, OK: The American Society of Mammologists.

Bi Z., Formenty P.B. \& Roth C.E. 2008. Hantavirus infection: a review and global update. The Journal of Infection in Developing Countries 2(01), 003-023. doi: 10.3855/jidc.317.

Bradley C.A. \& Altizer S. 2007. Urbanization and the ecology of wildlife diseases. Trends in Ecology e Evolution 22(2), 95-102. doi: 10.1016/j.tree.2006.11.001.

Bradley E.L. \& Terman C.R. 1981. A comparison of the adrenal histology, reproductive condition, and serum corticosterone concentrations of prairie deer mice (Peromyscus maniculatus bairdii) in captivity. Journal of Mammalogy 62(2), 353-361. doi: 10.2307/1380711.

Brearley G., Rhodes J., Bradley A., Baxter G., Seabrook L., Lunney D., Liu Y. \& McAlpine C. 2013. Wildlife disease prevalence in human-modified landscapes. Biological Reviews 88(2), 427-442. doi: 10.1111/brv.12009.

Buscha D.S. \& Hayward L.S. 2009. Stress in a conservation context: a discussion of glucocorticoid actions and how levels change with conservation-relevant variables. Biological Conservation 142(12), 2844-2853. doi: 10.1016/j. biocon.2009.08.013. 
Calisher C.H., Mills J.N., Root J.J., Doty J.B. \& Beaty B.J. 2011 . The relative abundance of deer mice with antibody to Sin Nombre virus corresponds to the occurrence of hantavirus pulmonary syndrome in nearby humans. VectorBorne and Zoonotic Diseases, 11(5), 577-582. doi: 10.1089/ vbz.2010.0122.

Childs J E., Ksiazek T.G., Spiropoulou C.F., Krebs J.W., Morzunov S., Maupin G.O., Gage K.L., Rollin P.E., Sarisky J. \& Enscore R.E. 1994. Serologic and genetic identification of Peromyscus maniculatus as the primary rodent reservoir for a new hantavirus in the southwestern United States. The Journal of Infectious Diseases 169(6), 1271-1280. doi: 10.1093/infdis/169.6.1271.

Cyr N.E. \& Romero L.M. 2009. Identifying hormonal habituation in field studies of stress. General and Comparative Endocrinology 161(3), 295-303. doi: 10.1016/j. ygcen.2009.02.001.

Dantzer B., Fletcher Q.E., Boonstra R. \& Sheriff M.J. 2014. Measures of physiological stress: a transparent or opaque window into the status, management and conservation of species? Conservation Physiology 2(1), cou023. doi: 10.1093/ conphys/cou023.

Douglass R.J., Calisher C.H., Wagoner K.D. \& Mills J.N. 2007. Sin Nombre virus infection of deer mice in Montana: characteristics of newly infected mice, incidence, and temporal pattern of infection. Journal of Wildlife Diseases 43(1), 12-22. doi: 10.7589/0090-3558-43.1.12.

Easterbrook J.D. \& Klein S.L. 2008. Immunological mechanisms mediating hantavirus persistence in rodent reservoirs. PLoS Pathog 4(11), el000172. doi: 10.1371/journal. ppat. 1000172.

Eleftheriou A., Palme R. \& Boonstra R. 2020. Assessment of the Stress Response in North American Deermice: Laboratory and Field Validation of Two Enzyme Immunoassays for Fecal Corticosterone Metabolites. Animals 10(7), 1120. doi: 10.3390/anil0071120

Fairbairn D.J. 1977. The spring decline in deer mice: death or dispersal? Canadian Journal of Zoology 55(1), 84-92. doi: 10.1139/z77-009.

Harper J.M. \& Austad S.N. 2001. Effect of capture and season on fecal glucocorticoid levels in deer mice (Peromyscus maniculatus) and red-backed voles (Clethrionomys gapperi). General and Comparative Endocrinology 123(3), 337-344. doi: 10.1006/gcen.2001.7682.

Hopkins A.S., Whitetail-Eagle J., Corneli A.L., Person B., Ettestad P.J., Dimenna M., Norstog J., Creswell J., Khan A.S. \& Olson J.G. 2002. Experimental evaluation of rodent exclusion methods to reduce hantavirus transmission to residents in a Native American community in New Mexico. Vector Borne and Zoonotic Diseases 2(2), 61-68. doi: 10.1089/153036602321131850.

Kuenzi A.J., Douglass R.J. \& Bond C.W. 2000. Sin Nombre virus in deer mice captured inside homes, southwestern Montana. Emerging Infectious Diseases 6(4), 386-388. doi: 10.3201/eid0604.000411.

Kuenzi A.J., Douglass R.J., White D. Jr., Bond C.W. \& Mills J.N. 2001. Antibody to Sin Nombre virus in rodents associated with peridomestic habitats in west central Montana. The American Journal of Tropical
Medicine and Hygiene 64(3-4), 137-146. doi: 10.4269/ ajtmh.2001.64.137.

Luis A.D., Douglass R.J., Hudson P.J., Mills J.N. \& Bjørnstad O.N. 2012. Sin Nombre hantavirus decreases survival of male deer mice. Oecologia 169(2), 431-439. doi: 10.1007/ s00442-011-2219-2.

Mills J.N., Yates T.L., Childs J.E., Parmenter R.R., Ksiazek T.G., Rollin P.E. \& C. Peters. 1995. Guidelines for working with rodents potentially infected with hantavirus. Journal of Mammalogy 76(3), 716-722. doi: 10.2307/1382742.

Mills J.N., Yates T.L., Ksiazek T.G., Peters C.J. \& Childs J.E. 1999. Long-term studies of hantavirus reservoir populations in the southwestern United States: rationale, potential, and methods. Emerging Infectious Diseases 5(1), 95-101. doi: 10.3201/eid0501.990111.

Millspaugh J.J. \& Washburn B.E. 2004. Use of fecal glucocorticoid metabolite measures in conservation biology research: considerations for application and interpretation. General and Comparative Endocrinology 138(3), 189-199. doi: 10.1016/j.ygcen.2004.07.002.

Mumby H.S., Mar K.U., Thitaram C., Courtiol A., Towiboon P., Min-Oo Z., Htut-Aung Y., Brown J.L. \& Lummaa V. 2015. Stress and body condition are associated with climate and demography in Asian elephants. Conservation Physiology 3(1), cov030. doi: 10.1093/conphys/cov030.

Pokharel S.S., Seshagiri P.B. \& Sukumar R. 2017. Assessment of season-dependent body condition scores in relation to fecal glucocorticoid metabolites in free-ranging Asian elephants. Conservation Physiology 5(1), cox039. doi: 10.1093/ conphys/cox039.

Rakotoniaina J.H., Kappeler P.M., Ravoniarimbinina P., Pechouskova E., Hämäläinen A.M., Grass J., Kirschbaum C. \& Kraus C. 2016. Does habitat disturbance affect stress, body condition and parasitism in two sympatric lemurs? Conservation Physiology 4(1), cow034. doi: 10.1093/ conphys/cow034.

R Development Core Team. 2009. R: a language and environment for statistical computing. Vienna, Austria: R Foundation for Statistical Computing. ISBN 3-900051-07-0. Accessed on the internet at http://www.R-project.org

Sarkar D. 2008. Lattice: multivariate data visualization with $R$. New York: Springer.

Schountz T., Calisher C.H., Richens T.R., Rich A.A., Doty J.B., Hughes M.T. \& Beaty B.J. 2007. Rapid field immunoassay for detecting antibody to Sin Nombre virus in deer mice. Emerging Infectious Diseases 13(10), 1604-1607. doi: 10.3201/eid1310.070356.

Touma C. \& Palme R. 2005. Measuring fecal glucocorticoid metabolites in mammals and birds: the importance of validation. Annals of the New York Academy of Sciences 1046(1), 54-74. doi: 10.1 196/annals.1343.006.

Ullman-Culleré M.H. \& Foltz C.J. 1999. Body condition scoring: a rapid and accurate method for assessing health status in mice. Comparative Medicine 49(3), 319-323.

Wickham H. 2009. ggplot2: elegant graphics for data analysis. New York: Springer-Verlag.

Wywialowski A. 1987. Habitat structure and predators: choices and consequences for rodent habitat specialists and generalists. Oecologia 72(1), 39-45. doi: 10.1007/BF00385042. 